\title{
Transmission Electron Microscopy
}

National Cancer Institute

\section{Source}

National Cancer Institute. Transmission Electron Microscopy. NCI Thesaurus. Code C18216.

An electron microscopy imaging technique that is utilized to examine structural components of a sample by passing electrons through the specimen. 